\title{
On the Structure of the Domain of a Symmetric Jump-type Dirichlet Form
}

\author{
by \\ René L. Schilling and Toshihiro Uemura
}

\begin{abstract}
We characterize the structure of the domain of a pure jump-type Dirichlet form which is given by a Beurling-Deny formula. In particular, we obtain sufficient conditions in terms of the jumping kernel guaranteeing that the test functions are a core for the Dirichlet form and that the form is a Silverstein extension. As an application we show that for recurrent Dirichlet forms the extended Dirichlet space can be interpreted in a natural way as a homogeneous Dirichlet space. For reflected Dirichlet spaces this leads to a simple purely analytic proof that the active reflected Dirichlet space (in the sense of Chen, Fukushima and Kuwae) coincides with the extended active reflected Dirichlet space.
\end{abstract}

2010 Mathematics Subject Classification: Primary 31C25, 60J45; Secondary 46E35. Keywords: jump-type Dirichlet form, locally shift-bounded kernel, Silverstein extension.

\section{$\S 1$. Introduction}

Let $\left(\varepsilon, C_{0}^{\infty}\left(\mathbb{R}^{d}\right)\right)$ be a closable Markovian (symmetric) form on the space $L^{2}\left(\mathbb{R}^{d}\right)=$ $L^{2}\left(\mathbb{R}^{d} ; d x\right)$, where $d x$ denotes Lebesgue measure on $\mathbb{R}^{d}$. It is well known that the closure $(\mathcal{E}, \mathcal{F})$ with respect to the norm $\sqrt{\mathcal{E}_{1}(u, u)}:=\left(\mathcal{E}(u, u)+\|u\|_{L^{2}}^{2}\right)^{1 / 2}$ is a regular Dirichlet form. On the other hand, on the set

$$
\mathcal{D}[\mathcal{E}]:=\left\{u \in L^{2}\left(\mathbb{R}^{d}\right): \mathcal{E}(u, u)<\infty\right\}
$$

the pair $(\mathcal{E}, \mathcal{D}[\mathcal{E}])$ becomes a (not necessarily regular) Dirichlet form on $L^{2}\left(\mathbb{R}^{d}\right)$.

It is a natural question to ask whether $\mathcal{F}$ and $\mathcal{D}[\mathcal{E}]$ coincide. If $\mathcal{E}(u, v)=$ $\frac{1}{2} \mathcal{D}(u, v)=\frac{1}{2}\langle\nabla u, \nabla v\rangle_{L^{2}}$ is the classical Dirichlet form, then it is known that

Communicated by T. Kumagai. Received September 29, 2010. Revised December 25, 2010.

R. L. Schilling: Institut für Mathematische Stochastik, Technische Universität Dresden, 01062 Dresden, Germany;

e-mail: rene.schilling@tu-dresden.de

T. Uemura: Department of Mathematics, Faculty of Engineering Science, Kansai University, Suita, Osaka, 564-8680 Japan;

e-mail: t-uemura@kansai-u.ac.jp

(C) 2012 Research Institute for Mathematical Sciences, Kyoto University. All rights reserved. 
$\mathcal{F}=W_{0}^{1,2}\left(\mathbb{R}^{d}\right)=\overline{C_{0}^{\infty}\left(\mathbb{R}^{d}\right)}\|\cdot\|_{1,2}$ whereas $\mathcal{D}[\mathcal{E}]=W^{1,2}\left(\mathbb{R}^{d}\right)$. Here $W^{1,2}\left(\mathbb{R}^{d}\right)$ denotes the usual $L^{2}$-Sobolev space of order 1 equipped with the norm $\|u\|_{1,2}:=\|\nabla u\|_{L^{2}}+$ $\|u\|_{L^{2}}$. Since $W^{1,2}\left(\mathbb{R}^{d}\right)=W_{0}^{1,2}\left(\mathbb{R}^{d}\right)$ (see e.g. Adams and Hedberg [AH] or Stein [St70]), we have $\mathcal{F}=\mathcal{D}[\mathcal{E}]$.

The identification of the domains has been studied for reflected Dirichlet forms using harmonic functions in [Ch92] or [Kuw02]. In this paper, we will discuss the problem for the jump-type form

$$
\mathcal{E}(u, v)=\frac{1}{2} \iint_{x \neq y}(u(x)-u(y))(v(x)-v(y)) N(d x, d y),
$$

for a measure $N(d x, d y)$. Since the expression under the integral is symmetric in $u$ and $v$, we can always assume that the measure $N(d x, d y)=N(d y, d x)$ is symmetric too. Recently one of us has investigated this problem for jump measures $N$ which have a symmetric density: $N(d x, d y)=k(x, y) d x d y$. Under a rather restrictive assumption on $k$, it is shown in [U07] that $\mathcal{F}=\mathcal{D}[\mathcal{E}]$. We will now extend this result to a more general setting.

The structure of the domain of a Dirichlet form has been studied for certain self-adjoint extensions or Markov extensions of the generator associated with the form (see e.g. [RZ, T96, KaT96]). In particular, it has been shown that for local Dirichlet forms and diffusion processes such extensions are trivial.

Our paper is organized as follows: In Section 2, we study the $L^{2}$-maximal domain and Silverstein extensions of the form (1.1); the homogeneous domain and reflected Dirichlet spaces are considered in Section 3, while the active reflected Dirichlet space is introduced in Section 4. The Appendix, Section 5, contains a brief survey on basic elements of the theory of Dirichlet forms.

\section{§2. $L^{2}$-maximal domains}

In this section, we formulate our setting and prove one of the main theorems.

Let $\mu(x, d y)$ be a positive kernel on $\mathbb{R}^{d} \times \mathcal{B}\left(\mathbb{R}^{d}\right)$ which generates on $\mathbb{R}^{d} \times \mathbb{R}^{d} \backslash \Delta$, $\Delta=\left\{(x, x): x \in \mathbb{R}^{d}\right\}$, a symmetric measure $N(d x, d y):=\mu(x, d y) d x ;$ see Remark 2 below for some comments on this assumption. Consider the following symmetric quadratic form $(\mathcal{E}, \mathcal{D}[\mathcal{E}])$ defined on $L^{2}\left(\mathbb{R}^{d}\right)$ :

$$
\left\{\begin{aligned}
\mathcal{E}(u, v) & :=\frac{1}{2} \iint_{x \neq y}(u(x)-u(y))(v(x)-v(y)) N(d x, d y), \\
\mathcal{D}[\mathcal{E}] & :=\left\{u \in L^{2}\left(\mathbb{R}^{d}, d x\right): \mathcal{E}(u, u)<\infty\right\} .
\end{aligned}\right.
$$

Set

$$
\Phi(x):=\int_{0<|x-y| \leq 1}|x-y|^{2} \mu(x, d y)=\int_{0<|h| \leq 1}|h|^{2} \mu(x, d h+x)
$$


and

$$
\Psi(x):=\int_{|x-y|>1} \mu(x, d y)=\int_{|h|>1} \mu(x, d h+x) .
$$

If $\Psi, \Phi \in L_{\text {loc }}^{1}\left(\mathbb{R}^{d}\right)$ Example 1.2.4 in [FOT94] shows that $\left(\mathcal{E}, C_{0}^{\infty}\left(\mathbb{R}^{d}\right)\right)$ is a closable Markovian (symmetric) form on $L^{2}\left(\mathbb{R}^{d}\right)$ and that the closure $(\mathcal{E}, \mathcal{F})$ is a regular (symmetric) Dirichlet form. The associated Markov process $\mathbf{M}$ is of pure jumptype.

The assumption that $\Psi, \Phi \in L_{\mathrm{loc}}^{1}\left(\mathbb{R}^{d}\right)$ is equivalent to saying that the test functions $C_{0}^{\infty}\left(\mathbb{R}^{d}\right)$ are contained in the form domain $\mathcal{D}[\mathcal{E}]$ (see Remark 2 at the end of this section).

For $x \in \mathbb{R}^{d}$ and $A \in \mathcal{B}\left(\mathbb{R}^{d}\right)$, put

$$
\nu(x, A):=\mu(x, A+x) .
$$

Obviously, $\nu$ defines again a kernel. Note that the jump kernel $\mu(x, B)$ represents the rate of jumps starting from $x$ and jumping into the set $B$, while $\nu(x, A)$ stands for the rate of jumps of size $A$ starting from $x$. With this convention we can rewrite the form in the following way: for $u \in \mathcal{F}$,

$$
\begin{aligned}
\mathcal{E}(u, v) & =\frac{1}{2} \iint_{x \neq y}(u(x)-u(y))(v(x)-v(y)) \mu(x, d y) d x \\
& =\frac{1}{2} \iint_{h \neq 0}(u(x)-u(x+h))(v(x)-v(x+h)) \mu(x, d h+x) d x \\
& =\frac{1}{2} \iint_{h \neq 0}(u(x)-u(x+h))(v(x)-v(x+h)) \nu(x, d h) d x .
\end{aligned}
$$

We will also need the concept of shift-bounded measures which is common in harmonic analysis (see e.g. $[\mathrm{BF}]$ ).

Definition 2.1 (locally shift-bounded kernel). A kernel $n(x, d y)$ defined on $\mathbb{R}^{d} \times$ $\mathcal{B}\left(\mathbb{R}^{d}\right)$ is said to be locally shift-bounded if there exists a constant $c>0$ such that

$$
n(x+z, A) \leq c n(x, A) \quad \text { for all } x, z \in \mathbb{R}^{d} \text { with }|z| \leq 1, A \subset B_{1}(0),
$$

where $B_{1}(0)$ is the open ball with centre 0 and radius 1 .

If $n(x, d y)$ is defined on $D \times \mathcal{B}(E)$ where $D, E \subset \mathbb{R}^{d}$ we call $n(x, d y)$ locally shift-bounded if the trivial extension $\bar{n}(x, d y)$ of $n(x, d y)$ onto $\mathbb{R}^{d} \times \mathcal{B}\left(\mathbb{R}^{d}\right)$,

$$
\bar{n}(x, B):= \begin{cases}n(x, B \cap E) & \text { if } x \in D, \\ 0 & \text { if } x \notin D,\end{cases}
$$

is locally shift-bounded. 
Examples of locally shift-bounded kernels are kernels which are absolutely continuous with respect to some measure $m$ on $\mathbb{R}^{d}$, say $n(x, d h)=n(x, h) m(d h)$, and where the density satisfies

$$
0<c:=\inf _{x \in \mathbb{R}^{d},|h| \leq 1} n(x, h) \leq \sup _{x \in \mathbb{R}^{d},|h| \leq 1} n(x, h)=: C<\infty
$$

Obviously,

$$
\frac{n(x+z, A)}{n(x, A)} \leq \frac{C m(A)}{c m(A)}=\frac{C}{c}<\infty \quad \text { for all } x, z \in B_{1}(0), A \subset B_{1}(0) .
$$

This is, for example, the case if $n(x, d h)$ does not depend on $x$ (i.e. if the underlying stochastic process is a Lévy process) or if the process is Lévy-like in the sense that $c \nu(h) \leq n(x, h) \leq C \nu(h)$ for all $|h| \leq 1$ and where $\nu(d h):=\nu(h) d h$ is the jump measure of some fixed Lévy process.

Let $\rho \in C_{0}^{\infty}\left(\mathbb{R}^{d}\right)$ be a smooth function satisfying $\rho(x)=\rho(-x) \geq 0, \operatorname{supp} \rho=$ $\overline{B_{1}(0)}$ and $\int \rho d x=1$. For $\varepsilon>0$, set $\rho_{\varepsilon}(\cdot):=\varepsilon^{-d} \rho(\cdot / \varepsilon)$. Clearly, $\rho_{\varepsilon} \geq 0$, supp $\rho_{\varepsilon}=$ $\overline{B_{1}(\varepsilon)}$ and $\int \rho_{\varepsilon} d x=1$. Denote by $J_{\varepsilon}[u]$ the Friedrichs mollifier, i.e.

$$
J_{\varepsilon}[u](x):=u \star \rho_{\varepsilon}(x)=\int u(x-z) \rho_{\varepsilon}(z) d z, \quad x \in \mathbb{R}^{d} .
$$

It is well known that $\left\|J_{\varepsilon}[u]\right\|_{L^{2}} \leq\|u\|_{L^{2}}$ and $\lim _{\varepsilon \rightarrow 0}\left\|J_{\varepsilon}[u]-u\right\|_{L^{2}}=0$ for all $u \in L^{2}$.

Lemma 2.2. Assume that $\Phi \in L_{\mathrm{loc}}^{1}\left(\mathbb{R}^{d}\right)$ and $\Psi \in L^{\infty}\left(\mathbb{R}^{d}\right)$ and that the kernel $\nu$ is locally shift-bounded. Then there exists a constant $c>0$ such that, for all $u \in \mathcal{D}[\mathcal{E}]$,

$$
\mathcal{E}\left(J_{\varepsilon}[u], J_{\varepsilon}[u]\right) \leq c \mathcal{E}(u, u)+4\|u\|_{L^{2}}^{2} \cdot\|\Psi\|_{L^{\infty}} .
$$

Proof. We split the integral of the form $\mathcal{E}\left(J_{\varepsilon}[u], J_{\varepsilon}[u]\right)$ into two parts:

$$
\begin{aligned}
\mathcal{E}\left(J_{\varepsilon}[u], J_{\varepsilon}[u]\right) & =\iint_{\mathbb{R}^{d} \times \mathbb{R}^{d}}\left(J_{\varepsilon}[u](x)-J_{\varepsilon}[u](y)\right)^{2} \mu(x, d y) d x \\
& \left.=\iiint_{|x-y| \leq 1}+\iint_{|x-y|>1}\right)\left(J_{\varepsilon}[u](x)-J_{\varepsilon}[u](y)\right)^{2} \mu(x, d y) d x \\
& =:(\mathrm{I})+(\mathrm{II}) .
\end{aligned}
$$

We will estimate the two expressions separately. Since $\rho_{\varepsilon}(z) d z$ is a probability measure, Jensen's inequality and Tonelli's theorem yield 


$$
\begin{aligned}
(\mathrm{I}) & =\iint_{|x-y| \leq 1}\left(\int(u(x-z)-u(y-z)) \rho_{\varepsilon}(z) d z\right)^{2} \mu(x, d y) d x \\
& \leq \int\left(\iint_{|x-y| \leq 1}(u(x-z)-u(y-z))^{2} \mu(x, d y) d x\right) \rho_{\varepsilon}(z) d z \\
& =\int\left(\iint_{|h| \leq 1}(u(x)-u(x+h))^{2} \mu(x+z, d h+x+z) d x\right) \rho_{\varepsilon}(z) d z \\
& =\int\left(\iint_{|h| \leq 1}(u(x)-u(x+h))^{2} \nu(x+z, d h) d x\right) \rho_{\varepsilon}(z) d z .
\end{aligned}
$$

Since $\operatorname{supp} \rho_{\varepsilon} \subset B_{1}(0)$ is compact and $\nu$ is locally shift-bounded we see that

$$
\begin{aligned}
(\mathrm{I}) & \leq c \int\left(\iint_{|h| \leq 1}(u(x)-u(x+h))^{2} \nu(x, d h) d x\right) \rho_{\varepsilon}(z) d z \\
& =c \iint_{|h| \leq 1}(u(x)-u(x+h))^{2} \nu(x, d h) d x \leq c \mathcal{E}(u, u) .
\end{aligned}
$$

As for the term (II), we see that

$$
\begin{aligned}
(\mathrm{II}) & \leq 2 \iint_{|x-y| \geq 1}\left(\left(J_{\varepsilon}[u](x)\right)^{2}+\left(J_{\varepsilon}[u](y)\right)^{2}\right) \mu(x, d y) d x \\
& =4 \int\left(J_{\varepsilon}[u](x)\right)^{2}\left(\int_{|x-y| \geq 1} \mu(x, d y)\right) d x \\
& \leq 4\|\Psi\|_{L^{\infty}} \int\left(J_{\varepsilon}[u](x)\right)^{2} d x=4\|\Psi\|_{L^{\infty}} \cdot\left\|J_{\varepsilon}[u]\right\|_{L^{2}}^{2} \leq 4\|\Psi\|_{L^{\infty}} \cdot\|u\|_{L^{2}}^{2} .
\end{aligned}
$$

If we combine both calculations, our claim follows.

Remark. With some simple modifications in the proof of Lemma 2.2 we can show that $\mathcal{E}\left(J_{\varepsilon}[u], J_{\varepsilon}[u]\right) \leq c(K) \mathcal{E}(u, u)$ for all $u \in \mathcal{F}$ with $\operatorname{supp} u \subset K$ for some fixed compact set $K$. In this case, we do not need to split the integral into two parts (I) $+($ II), but we can estimate the whole expression directly. This means that we only have to assume $\Phi, \Psi \in L_{\text {loc }}^{1}$.

If we require that (2.4) holds for all $A \subset \mathbb{R}^{d}$ and not just for $A \subset B_{1}(0)$, the above modification of the proof of Lemma 2.2 gives $\mathcal{E}\left(J_{\varepsilon}[u], J_{\varepsilon}[u]\right) \leq c \mathcal{E}(u, u)$ for all $u \in \mathcal{F}$. Again, we only need to assume that $\Phi, \Psi \in L_{\mathrm{loc}}^{1}$.

Lemma 2.3. For $\varepsilon>0$, define a real function $T_{\varepsilon}$ as follows:

$$
T_{\varepsilon}(x):=(-1 / \varepsilon) \vee(x-(-\varepsilon) \vee x \wedge \varepsilon) \wedge 1 / \varepsilon, \quad x \in \mathbb{R} .
$$

Then $T_{\varepsilon}$ is a normal contraction, i.e. $T_{\varepsilon}$ satisfies

$$
\left|T_{\varepsilon}(x)\right| \leq|x| \text { and }\left|T_{\varepsilon}(x)-T_{\varepsilon}(y)\right| \leq|x-y|, \quad x, y \in \mathbb{R} .
$$

Moreover, for any $x \in \mathbb{R}, T_{\varepsilon}(x)$ converges to $x$ as $\varepsilon \rightarrow 0$. 
Proof. The second assertion is obvious from the definition of $T_{\varepsilon}$. So we show the first assertion. Let $f(x)=x$ and take $\varepsilon>0$. Then it is easy to see that the functions $f_{\varepsilon}:=(-\varepsilon) \vee f \wedge \varepsilon$ and $f-f_{\varepsilon}$ are normal contractions. Hence the lemma follows from the fact that the composition of two normal contractions is again a normal contraction.

We can now show the main result of this section.

Theorem 2.4. Assume that $\Phi \in L_{\mathrm{loc}}^{1}\left(\mathbb{R}^{d}\right)$ and $\Psi \in L^{\infty}\left(\mathbb{R}^{d}\right)$ hold and that the kernel $\nu$ is locally shift-bounded. Then

$$
\mathcal{D}[\mathcal{E}]=\left\{u \in L^{2}\left(\mathbb{R}^{d}\right): \mathcal{E}(u, u)<\infty\right\}=\mathcal{F} ;
$$

this means that every element from $\mathcal{D}[\mathcal{E}]$ can be approximated by a sequence of functions in $C_{0}^{\infty}\left(\mathbb{R}^{d}\right)$ with respect to $\mathcal{E}_{1}$.

Proof. For $u \in \mathcal{D}[\mathcal{E}]$ we define the Friedrichs mollifier $J_{\varepsilon}[u]=u \star \rho_{\varepsilon}$ as in (2.5). Note that $J_{\varepsilon}[u] \in C_{\infty}\left(\mathbb{R}^{d}\right)$ is a continuous function vanishing at infinity. Indeed, for the Fourier transform $\mathscr{F} f$ we know that $\mathscr{F} u \in L^{2}\left(\mathbb{R}^{d}\right)$ and $\mathscr{F} \rho_{\varepsilon} \in \mathscr{S} \subset L^{2}\left(\mathbb{R}^{d}\right)$; here $\mathscr{S}$ denotes the Schwartz space of rapidly decreasing functions. By the convolution theorem

$$
\mathscr{F}\left(J_{\varepsilon}[u]\right)=\mathscr{F}\left(u \star \rho_{\varepsilon}\right)=\mathscr{F} u \cdot \mathscr{F} \rho_{\varepsilon} \in L^{1}\left(\mathbb{R}^{d}\right)
$$

and the Riemann-Lebesgue lemma tells us that $J_{\varepsilon}[u]=\mathscr{F}-1 \mathscr{F}\left(J_{\varepsilon}[u]\right) \in C_{\infty}\left(\mathbb{R}^{d}\right)$.

Since $J_{\varepsilon}[u]$ vanishes at infinity, $T_{\varepsilon}\left(J_{\varepsilon}[u]\right)$ has compact support, and therefore $w_{\varepsilon}:=J_{\varepsilon}\left[T_{\varepsilon}\left(J_{\varepsilon}[u]\right)\right] \in C_{0}^{\infty}\left(\mathbb{R}^{d}\right) \subset \mathcal{F}$. Moreover, $L^{2}-\lim _{\varepsilon \rightarrow 0} w_{\varepsilon}=u$ since

$$
\begin{aligned}
\left\|w_{\varepsilon}-u\right\|_{L^{2}} & \leq\left\|J_{\varepsilon}\left[T_{\varepsilon}\left(J_{\varepsilon}[u]\right)\right]-J_{\varepsilon}\left[T_{\varepsilon}(u)\right]\right\|_{L^{2}}+\left\|J_{\varepsilon}\left[T_{\varepsilon}(u)\right]-J_{\varepsilon}[u]\right\|_{L^{2}}+\left\|J_{\varepsilon}[u]-u\right\|_{L^{2}} \\
& \leq\left\|T_{\varepsilon}\left(J_{\varepsilon}[u]\right)-T_{\varepsilon}(u)\right\|_{L^{2}}+\left\|T_{\varepsilon}(u)-u\right\|_{L^{2}}+\left\|J_{\varepsilon}[u]-u\right\|_{L^{2}} \\
& \leq 2\left\|J_{\varepsilon}[u]-u\right\|_{L^{2}}+\left\|T_{\varepsilon}(u)-u\right\|_{L^{2}} \stackrel{\varepsilon \rightarrow 0}{\longrightarrow} 0 .
\end{aligned}
$$

The second estimate follows from Jensen's inequality since $\rho_{\varepsilon}(z) d z$ is a probability measure, while the third inequality results from the normal contraction property of $T_{\varepsilon}$. For the limits we use the fact that the Friedrichs mollifier converges to $u$ in $L^{2}$ and, for the second expression, we use the dominated convergence theorem.

According to Lemma 2.2 we see

$$
\mathcal{E}\left(w_{\varepsilon}, w_{\varepsilon}\right) \leq c \mathcal{E}\left(T_{\varepsilon}\left(J_{\varepsilon}[u]\right), T_{\varepsilon}\left(J_{\varepsilon}[u]\right)\right)+4\left\|T_{\varepsilon}\left(J_{\varepsilon}[u]\right)\right\|_{L^{2}}^{2}\|\Psi\|_{L^{\infty}} .
$$

Since $T_{\varepsilon}$ is a normal contraction, we get

$$
\mathcal{E}\left(T_{\varepsilon}\left(J_{\varepsilon}[u]\right), T_{\varepsilon}\left(J_{\varepsilon}[u]\right)\right) \leq \mathcal{E}\left(J_{\varepsilon}[u], J_{\varepsilon}[u]\right)
$$


and

$$
\left\|T_{\varepsilon}\left(J_{\varepsilon}[u]\right)\right\|_{L^{2}} \leq\left\|J_{\varepsilon}[u]\right\|_{L^{2}} \leq\|u\|_{L^{2}} .
$$

A further application of Lemma 2.2 shows

$$
\mathcal{E}\left(w_{\varepsilon}, w_{\varepsilon}\right) \leq c\left(c \mathcal{E}(u, u)+4\|u\|_{L^{2}}^{2}\|\Psi\|_{L^{\infty}}\right)+4\|u\|_{L^{2}}^{2}\|\Psi\|_{L^{\infty}} .
$$

This means that the family $\left\{\mathcal{E}\left(w_{\varepsilon}, w_{\varepsilon}\right)\right\}_{\varepsilon>0}$, hence $\left\{\mathcal{E}_{1}\left(w_{\varepsilon}, w_{\varepsilon}\right)\right\}_{\varepsilon>0}$, is uniformly bounded. Therefore, we can use the Banach-Saks theorem to deduce that for a subsequence $\{\varepsilon(n)\}_{n \in \mathbb{N}}$ with $\lim _{n \rightarrow \infty} \varepsilon(n)=0$ the Cesàro means

$$
\frac{1}{n} \sum_{k=1}^{n} w_{\varepsilon(k)}
$$

converge to a function $\tilde{u} \in \mathcal{F}$ with respect to $\sqrt{\mathcal{E}_{1}}$ and, in particular, in $L^{2}$. On the other hand, we know that $w_{\varepsilon}$, hence any subsequence and any convex combination, converges to $u \in L^{2}\left(\mathbb{R}^{d}\right)$. Since $L^{2}$-limits are unique, we conclude that $u=\tilde{u} \in \mathcal{F}$.

Recall that a symmetric Dirichlet form $(\eta, \mathcal{D}[\eta])$ on $L^{2}\left(\mathbb{R}^{d}\right)$ is said to be an extension of the Dirichlet form $(\mathcal{E}, \mathcal{F})$ if $\mathcal{D}[\eta] \supset \mathcal{F}$ and if $\eta(u, u)=\mathcal{E}(u, u)$ whenever $u \in \mathcal{F}$. By $\mathcal{A}(\mathcal{E}, \mathcal{F})$ we denote the family of all possible extensions of the form $(\mathcal{E}, \mathcal{F})$. Clearly, $(\mathcal{E}, \mathcal{D}[\mathcal{E}]) \in \mathcal{A}(\mathcal{E}, \mathcal{F})$. An element $(\eta, \mathcal{D}[\eta])$ of $\mathcal{A}(\mathcal{E}, \mathcal{F})$ is called a Silverstein extension if $\mathcal{F}_{b}$ is an algebraic ideal in $\mathcal{D}[\eta]_{b}$. (The subscript $b$ indicates that we consider only bounded elements of the respective set.) Most papers dealing with Silverstein extensions of Dirichlet forms consider only local Dirichlet forms (see e.g. [T96]).

The following theorem is, in an abstract setting of regular Dirichlet forms, contained in Kuwae [Kuw02, §5]. Since that paper is quite technical, we give a very short alternative proof based on our techniques. Note that our assumptions entail regularity.

Theorem 2.5. Assume that $\Phi \in L_{\mathrm{loc}}^{1}\left(\mathbb{R}^{d}\right)$ and $\Psi \in L_{\mathrm{loc}}^{\infty}\left(\mathbb{R}^{d}\right)$ and that the kernel $\nu$ is locally shift-bounded. Then the Dirichlet form $(\mathcal{E}, \mathcal{D}[\mathcal{E}])$ is a Silverstein extension of the form $(\mathcal{E}, \mathcal{F})$, i.e. $\mathcal{F}_{b}$ is an ideal in $\mathcal{D}[\mathcal{E}]_{b}$.

Proof. As in [U07], it is enough to show that $u \cdot \varphi \in \mathcal{F}_{b}$ whenever $u \in \mathcal{D}[\mathcal{E}]_{b}$ and $\varphi \in C_{0}^{\infty}\left(\mathbb{R}^{d}\right)$. Using the fact that $u$ is bounded and $\varphi$ is compactly supported, we see that $u \varphi$ belongs to $L^{2}\left(\mathbb{R}^{d}\right), J_{\varepsilon}[u \varphi]$ belongs to $C_{0}^{\infty}\left(\mathbb{R}^{d}\right) \subset \mathcal{F}$ and $J_{\varepsilon}[u \varphi]$ converges to $u \varphi$ in $L^{2}\left(\mathbb{R}^{d}\right)$. As before $J_{\varepsilon}[u \varphi]=(u \varphi) \star \rho_{\varepsilon}$ is the Friedrichs mollifier.

We will prove that the family $\left\{\mathcal{E}\left(J_{\varepsilon}[u \varphi], J_{\varepsilon}[u \varphi]\right)\right\}_{0<\varepsilon<1}$ is uniformly bounded. Indeed, let us denote by $K$ the compact support of $\varphi, K:=\operatorname{supp} \varphi$. Then using an 
estimate similar to the one in Lemma 2.2 and putting $K_{1}:=\left\{x+y \in \mathbb{R}^{d}: x \in K\right.$, $|y|<1\}$, we see that

$$
\mathcal{E}\left(J_{\mathcal{\varepsilon}}[u \varphi], J_{\varepsilon}[u \varphi]\right) \leq c \mathcal{E}(u \varphi, u \varphi)+4\|u\|_{L^{\infty}}^{2}\|\varphi\|_{L^{2}}^{2}\|\Psi\|_{L^{\infty}\left(K_{1}\right)}
$$

and

$$
\begin{aligned}
\mathcal{E}(u \varphi, u \varphi)= & \iint_{x \neq y}(u(x) \varphi(x)-u(y) \varphi(y))^{2} \mu(x, d y) d x \\
\leq & 2 \iint_{x \neq y} u(x)^{2}(\varphi(x)-\varphi(y))^{2} \mu(x, d y) d x \\
& +2 \iint_{x \neq y} \varphi(y)^{2}(u(x)-u(y))^{2} \mu(x, d y) d x \\
\leq & 2\|u\|_{L^{\infty}}^{2} \mathcal{E}(\varphi, \varphi)+2\|\varphi\|_{L^{\infty}}^{2} \mathcal{E}(u, u) .
\end{aligned}
$$

Since $\left\{\mathcal{E}\left(J_{\varepsilon}[u \varphi], J_{\varepsilon}[u \varphi]\right)\right\}_{0<\varepsilon<1}$ is uniformly bounded, we may argue as in the second part of the proof of Theorem 2.4 and take a subsequence of $\left\{J_{\varepsilon(k)}[u \varphi]\right\}_{k \in \mathbb{N}}$, for which $\varepsilon(k)$ goes to 0 as $k \rightarrow \infty$, such that the Cesàro means converge in $\sqrt{\mathcal{E}_{1}}$ to some $v \in \mathcal{F}$. Because of the uniqueness of $L^{2}$-limits $v=u \varphi$ and the proof is then complete.

We will now consider the case where the Dirichlet form is defined on an arbitrary open set $D \subset \mathbb{R}^{d}$. By $\lambda_{D}$ we denote Lebesgue measure on $D$ and we assume that $N_{D}(d x, d y):=\mu_{D}(x, d y) \lambda_{D}(d x)$ is a symmetric measure on $D \times D \backslash \Delta$. Set

$$
\left\{\begin{aligned}
\mathcal{E}_{D}(u, v) & :=\iint_{D \times D \backslash \Delta}(u(x)-u(y))((x)-v(y)) \mu(x, d y) \lambda_{D}(d x), \\
\mathcal{D}\left[\mathcal{E}_{D}\right] & :=\left\{u \in L^{2}\left(D, \lambda_{D}\right): \varepsilon_{D}(u, u)<\infty\right\} .
\end{aligned}\right.
$$

Define, with the obvious changes, $\Phi$ and $\Psi$ as in (2.1) and (2.2). If $\Phi, \Psi \in L_{\mathrm{loc}}^{1}(D)$, then $\left(\mathcal{E}_{D}, \mathcal{D}\left[\mathcal{E}_{D}\right]\right)$ is a Dirichlet form on $L^{2}\left(D, \lambda_{D}\right)\left(=L^{2}\left(\bar{D}, \lambda_{D}\right)\right)$. As usual, $\bar{D}$ is the closure of $D, C_{0}^{\infty}(D)=\left\{u \in C_{0}^{\infty}\left(\mathbb{R}^{d}\right): \operatorname{supp} u \subset D\right\}$, and $C_{0}^{\infty}(\bar{D})=\left\{\left.u\right|_{\bar{D}}\right.$ : $\left.u \in C^{\infty}\left(\mathbb{R}^{d}\right)\right\}$.

We write $\mathcal{F}_{\bar{D}}\left(\right.$ resp. $\left.\mathcal{F}_{D}^{0}\right)$ for the closure of $C_{0}^{\infty}(\bar{D})$ (resp. $C_{0}^{\infty}(D)$ ) with respect to $\mathcal{E}_{D, 1}(u):=\mathcal{E}_{D}(u, u)+\|u\|_{L^{2}(D)}^{2}$. Clearly, $\mathcal{F}_{\bar{D}}, \mathcal{F}_{D}^{0} \subset \mathcal{D}\left[\mathcal{E}_{D}\right]$ and $\left(\mathcal{E}_{D}, \mathcal{F}_{\bar{D}}\right)$ (resp. $\left.\left(\varepsilon_{D}^{0}, \mathcal{F}_{D}^{0}\right)\right)$ are regular symmetric Dirichlet forms on $L^{2}\left(\bar{D}, \lambda_{D}\right)\left(\right.$ resp. $L^{2}\left(D, \lambda_{D}\right)$ ), where $\mathcal{E}_{D}^{0}$ denotes the restriction of $\mathcal{E}_{D}$ to $\mathcal{F}_{D}^{0} \times \mathcal{F}_{D}^{0}$. Moreover, according to Theorem 4.4.3 in [FOT94], we have the identity

$$
\mathcal{F}_{D}^{0}=\left\{u \in \mathcal{F}_{\bar{D}}: \tilde{u}=0, \mathcal{E}_{D} \text {-quasi everywhere on } \partial D\right\},
$$

where $\tilde{u}$ denotes the $\mathcal{E}_{D}$-quasi-continuous modification of $u \in \mathcal{F}_{\bar{D}}$. 
We are interested in the relation of $\mathcal{D}\left[\mathcal{E}_{D}\right]$ and $\mathcal{F}_{\bar{D}}$. For this, we extend a function $u$ defined on $D$ to the whole space $\mathbb{R}^{d}$ by setting $u=0$ on $\mathbb{R}^{d} \backslash D$.

Proposition 2.6. Let $\left(\mathcal{E}_{D}, \mathcal{D}\left[\mathcal{E}_{D}\right]\right)$ be as in (2.6) where $D \subset \mathbb{R}^{d}$ is an open set. Assume that $\Phi \in L_{\mathrm{loc}}^{1}(D)$ and $\Psi \in L^{\infty}(D)$ hold and that the kernel $\nu(x, A):=$ $\mu(x, A+x), x \in D, B \in \mathcal{B}(D)$, is locally shift-bounded. Then

$$
\mathcal{D}\left[\mathcal{E}_{D}\right]:=\left\{u \in L^{2}\left(D, \lambda_{D}\right): \mathcal{E}_{D}(u, u)<\infty\right\}=\mathcal{F}_{\bar{D}} .
$$

Proof. Note that $\mathcal{D}\left[\mathcal{E}_{D}\right]=\left\{u \in L^{2}\left(\bar{D}, \lambda_{D}\right): \mathcal{E}_{D}(u, u)<\infty\right\}$ since $L^{2}\left(D ; \lambda_{D}\right)=$ $L^{2}\left(\bar{D} ; \lambda_{D}\right)$. Let $u \in \mathcal{D}\left[\mathcal{E}_{D}\right]$ and extend $u$ by zero outside of $\bar{D}$; in particular $u \in$ $L^{2}\left(\mathbb{R}^{d}\right)$. Consider the mollifier $J_{\varepsilon}[u]$ as in (2.5). As in the proof of Theorem 2.4, we see $w_{\varepsilon}=J_{\varepsilon}\left[T_{\varepsilon}\left(J_{\varepsilon}[u]\right)\right] \in C_{0}^{\infty}\left(\mathbb{R}^{d}\right)$, hence $\left.w_{\varepsilon}\right|_{\bar{D}} \in C_{0}^{\infty}(\bar{D})$. We also see that $\left.w_{\varepsilon}\right|_{\bar{D}}$ converges to $u$ in $L^{2}\left(D, \lambda_{D}\right)$ and the family $\left\{\varepsilon_{D}\left(\left.w_{\varepsilon}\right|_{\bar{D}},\left.w_{\varepsilon}\right|_{\bar{D}}\right)\right\}_{0<\varepsilon<1}$ is uniformly bounded. The remaining part of the proof is now exactly as in Theorem 2.4.

Example 2.7 (Censored stable process in an open set; [BBC03]). Let $0<\alpha<2$ and $\mu(x, d y)=c_{d, \alpha}|x-y|^{-d-\alpha} d y$ where $c_{d, \alpha}$ is a positive constant depending on $d$ and $\alpha$. In this case, the assumptions of Proposition 2.6 are satisfied. Therefore,

$$
\left\{\begin{aligned}
\mathcal{E}_{D}(u, v) & =c_{d, \alpha} \iint_{D \times D \backslash \Delta} \frac{(u(x)-u(y))(v(x)-v(y))}{|x-y|^{d+\alpha}} d x d y \\
\mathcal{D}\left[\mathcal{E}_{D}\right] & =\left\{u \in L^{2}(D): \mathcal{E}_{D}(u, u)<\infty\right\}
\end{aligned}\right.
$$

is a regular Dirichlet form on $L^{2}(\bar{D})$. The corresponding stochastic process is called a censored stable process on $D$ and $\mathcal{D}\left[\mathcal{E}_{D}\right]$ is also obtained by taking the $\mathcal{E}_{D, 1}$-closure of $C_{0}^{\infty}(\bar{D})$. Moreover $\mathcal{D}\left[\mathcal{E}_{D}\right]$ is the active reflected Dirichlet space (see Section 4).

We conclude this section with a few remarks on our assumptions on $N(d x, d y)$.

Remark. Often the Dirichlet form $\mathcal{E}$ is given in terms of its Beurling-Deny representation (1.1) with a jump kernel $N(d x, d y)$ which is not necessarily given as $\mu(x, d y) d x$. This is, e.g., the case when we start with a stochastic process admitting a Lévy system. Since the integrand in (1.1) is symmetric, we can always assume that $N(d x, d y)$ is symmetric. In order to make (1.1) convergent, one usually requires that

$$
\iint_{K \times K}|x-y|^{2} N(d x, d y)+\iint_{K \times L^{c}} N(d x, d y)<\infty, \quad \begin{array}{ll}
\text { for all } K \subset \stackrel{\circ}{L} \subset \mathbb{R}^{d} \\
K, L \text { compact. }
\end{array}
$$

Obviously, this is equivalent to saying that

$$
\iint_{K \times \mathbb{R}^{d}}\left(|x-y|^{2} \wedge 1\right) N(d x, d y)<\infty \quad \text { for all compact sets } K \subset \mathbb{R}^{d} .
$$


Consider now the finite measure $M(d x, d y):=\left(|x-y|^{2} \wedge 1\right) N(d x, d y)$ and set

$$
m(K):=M\left(K \times \mathbb{R}^{d}\right)=\iint_{K \times \mathbb{R}^{d}}\left(|x-y|^{2} \wedge 1\right) N(d x, d y), \quad K \subset \mathbb{R}^{d} \text { compact. }
$$

By a standard technique (cf. [EK, Appendix 8] or [K, Chapter 5]), we can disintegrate the bi-measure $M$ and find

$$
M(d x, d y)=\mu(x, d y) m(d x) \text { and, by symmetry, } M(d x, d y)=\mu(y, d x) m(d y) .
$$

Thus,

$$
N(d x, d y)=\frac{1}{|x-y|^{2} \wedge 1} \mu(x, d y) m(d x)
$$

and we have $m(d x) \ll d x$ - which allows us to use Lebesgue measure as reference measure -if, and only if, $M\left(d x \times \mathbb{R}^{d}\right) \ll d x$, i.e. if

$\operatorname{Leb}(X)=0 \Rightarrow \iint_{(K \cap X) \times \mathbb{R}^{d}}\left(|x-y|^{2} \wedge 1\right) N(d x, d y)=0$ for all compact $K \subset \mathbb{R}^{d}$.

This is clearly equivalent to saying that $N\left((K \cap X) \times \mathbb{R}^{d}\right)=0$ for all compact sets $K \subset \mathbb{R}^{d}$.

Finally, (2.8) or (2.7) is the same as $\Phi, \Psi \in L_{\text {loc }}^{1}$ since

$$
\begin{aligned}
\Phi(x)+\Psi(x) & =\int_{|x-y| \leq 1}|x-y|^{2} \mu(x, d y)+\int_{|x-y|>1} \mu(x, d y) \\
& =\int_{\mathbb{R}^{d}}\left(|x-y|^{2} \wedge 1\right) \mu(x, d y) .
\end{aligned}
$$

In particular, $C_{0}^{\infty}\left(\mathbb{R}^{d}\right) \subset \mathcal{D}[\mathcal{E}]$. Conversely, if $C_{0}^{\infty}\left(\mathbb{R}^{d}\right) \subset \mathcal{D}[\mathcal{E}]$, we fix any two compact sets $K \subset \stackrel{\circ}{L}$ and we pick $\chi \in C_{0}^{\infty}\left(\mathbb{R}^{d}\right)$ such that $\mathbb{1}_{K} \leq \chi(x) \leq \mathbb{1}_{L}$. Since the functions $u_{j}(x):=x_{j} \chi(x), j=1, \ldots, d$, are of class $C_{0}^{\infty}\left(\mathbb{R}^{d}\right)$, and since $\sum_{j=1}^{d} \varepsilon\left(u_{j}, u_{j}\right)+\varepsilon(\chi, \chi)<\infty$, it is easy to deduce (2.7).

\section{§3. Homogeneous domains and reflected Dirichlet spaces}

In analogy to (homogeneous) Sobolev spaces we call

$$
\dot{\mathcal{D}}[\mathcal{E}]:=\left\{u: \mathbb{R}^{d} \stackrel{\text { measurable }}{\longrightarrow} \mathbb{R}: \mathcal{E}(u, u)<\infty\right\}
$$

the homogeneous domain (see [U07]). Strictly speaking, the symbol $\mathcal{E}$ appearing on the right hand side is an extension of the original form. Here we do not need to stress this fact since $\mathcal{E}$ is pure-jump given by the integral expression (1.1) which is a priori defined on all measurable functions. It is well known that for the extended Dirichlet space $\mathcal{F}_{e}$ (cf. [FOT94]),

$$
\mathcal{F}=\mathcal{F}_{e} \cap L^{2}\left(\mathbb{R}^{d}\right) \subset \mathcal{F}_{e} \subset \dot{\mathcal{D}}[\mathcal{E}] .
$$


In general, it is not clear whether $\dot{\mathcal{D}}[\mathcal{E}]$ coincides with $\mathcal{F}_{e}$. In [U07], where we assumed the existence of a jump density, we obtained a restrictive sufficient condition for $\dot{\mathcal{D}}[\mathcal{E}]=\mathcal{F}_{e}$ in terms of the density; this condition also entailed that the form is recurrent. In the present context we can give a more practical and more relaxed condition on jump kernels.

We begin with a simple lemma which holds for general Dirichlet forms.

Lemma 3.1. Let $X$ be a locally compact separable metric space and $m$ a positive Radon measure on $X$ with full support. Assume that $(\mathfrak{q}, \mathcal{Q})$ is a regular symmetric Dirichlet form on $L^{2}(X ; m)$ which is recurrent. Then there exists a decreasing sequence $\left(U^{\ell}\right)_{\ell \in \mathbb{N}}$ of open sets with $m\left(U^{\ell}\right)<1 / \ell$ and a sequence $\left\{\varphi_{n}\right\}_{n \in \mathbb{N}} \subset$ $C_{0}(X) \cap \mathcal{Q}$ so that $\sup _{n \in \mathbb{N}} \mathfrak{q}\left(\varphi_{n}, \varphi_{n}\right)<\infty, 0 \leq \varphi_{n}(x) \leq 1$ for all $x$, and $\varphi_{n}$ converges to 1 uniformly on all sets of the form $K \backslash U^{\ell}$ where $\ell \in \mathbb{N}$ and $K \subset X$ is compact.

Proof. Since $(\mathfrak{q}, \mathcal{Q})$ is recurrent, we can find a sequence $\left\{\psi_{n}\right\}_{n \in \mathbb{N}} \subset \mathcal{Q}$ so that

$$
\lim _{n \rightarrow \infty} \psi_{n}=1 \text { a.e. and } \lim _{n \rightarrow \infty} \mathfrak{q}\left(\psi_{n}, \psi_{n}\right)=0
$$

(see Theorem 1.6.3 in [FOT94]). Denote the exceptional set by $N$. Since $m$ is a Radon measure, there exists a decreasing sequence of open sets $U^{\ell} \supset N$ such that $m\left(U^{\ell}\right)<1 / \ell$. Because of the regularity of the Dirichlet form we can assume that the sequence $\left\{\psi_{n}\right\}_{n \in \mathbb{N}}$ is actually from $C_{0}(X) \cap \mathcal{Q}$. Moreover, we can assume that $0 \leq \psi_{n} \leq 1$; otherwise we replace $\psi_{n}$ by $0 \vee \psi_{n} \wedge 1$ and remark that normal contractions operate on the Dirichlet space $(\mathcal{Q}, \mathfrak{q})$. As $\lim _{n \rightarrow \infty} \mathfrak{q}\left(\psi_{n}, \psi_{n}\right)=0$, we can extract a subsequence $\left\{\psi_{n(k)}\right\}_{k \in \mathbb{N}}$ satisfying $\mathfrak{q}\left(\psi_{n(k)}, \psi_{n(k)}\right)<2^{-2 k}$ for each $k$. For $k \geq 1$, we define

$$
\varphi_{k}:=\left(\psi_{n(1)}+\cdots+\psi_{n(k)}\right) \wedge 1 .
$$

Then $\varphi_{k} \in C(X) \cap \mathcal{Q}, 0 \leq \varphi_{k} \leq 1$ and $\sup _{k \in \mathbb{N}} \varphi_{k}=1$. Moreover,

$$
\begin{aligned}
\mathfrak{q}\left(\varphi_{k}, \varphi_{k}\right) & \leq \mathfrak{q}\left(\psi_{n(1)}+\cdots+\psi_{n(k)}, \psi_{n(1)}+\cdots+\psi_{n(k)}\right) \\
& \leq \sum_{\ell=1}^{k} 2^{\ell} \mathfrak{q}\left(\psi_{n(\ell)}, \psi_{n(\ell)}\right) \leq \sum_{\ell=1}^{k} 2^{-\ell}<\infty
\end{aligned}
$$

where we used the contraction property of Dirichlet forms and, repeatedly, the estimate $\mathfrak{q}(f+g, f+g) \leq 2 \mathfrak{q}(f, f)+2 \mathfrak{q}(g, g)$. Since $\left\{1-\varphi_{k} \mathbb{1}_{X \backslash U^{\ell}}\right\}_{k \in \mathbb{N}}$ is a decreasing sequence of upper semicontinuous functions with limit 0 , we can use Dini's theorem (cf. [R, pp. 195-196]) to conclude that the convergence $\varphi_{k} \stackrel{k \rightarrow \infty}{\longrightarrow} 1$ is uniform on all sets of the form $K \backslash U^{\ell}$ where $K \subset X$ is compact. 
Theorem 3.2. Assume that $\Phi \in L_{\text {loc }}^{1}\left(\mathbb{R}^{d}\right)$ and $\Psi \in L^{\infty}\left(\mathbb{R}^{d}\right)$ and that the kernel $\nu$ is locally shift-bounded. Assume further that the Dirichlet form $(\mathcal{E}, \mathcal{F})$ is recurrent. Then the homogeneous domain $\dot{\mathcal{D}}[\mathcal{E}]$ coincides with the extended Dirichlet space $\mathcal{F}_{e}$.

Proof. Let $\left(U^{\ell}\right)_{\ell \in \mathbb{N}}$ be the decreasing sequence of open sets from the lemma above. Denote $V^{\ell}:=X \backslash U^{\ell}$. Note that $\dot{\mathcal{D}}[\mathcal{E}]_{b}=\left\{u: \mathbb{R}^{d} \stackrel{\text { m'ble }}{\longrightarrow} \mathbb{R}: \mathcal{E}(u, u)<\infty\right\} \cap L^{\infty}\left(\mathbb{R}^{d}\right)$ is dense in $\dot{\mathcal{D}}[\mathcal{E}]$ with respect to the seminorm $\sqrt{\mathcal{E}}$.

Fix $u \in \dot{\mathcal{D}}[\mathcal{E}]_{b}$. It is not hard to see that $\varphi \cdot u \in \dot{\mathcal{D}}[\mathcal{E}]_{b} \cap L^{2}\left(\mathbb{R}^{d}\right)$ for all $\varphi \in C_{0}^{\infty}\left(\mathbb{R}^{d}\right)$. From Theorem 2.4 we know that $u \cdot \varphi \in \mathcal{F}=\mathcal{D}[\mathcal{E}]$.

Since the form $(\mathcal{E}, \mathcal{F})$ is recurrent, Lemma 3.1 guarantees the existence of a nonnegative and bounded sequence $\left\{\varphi_{n}\right\}_{n \in \mathbb{N}} \subset C_{0}^{\infty}\left(\mathbb{R}^{d}\right)$ such that $\varphi_{n}$ converges to 1 uniformly on compact sets in $V^{\ell}$ and $\sup _{n \in \mathbb{N}} \mathcal{E}\left(\varphi_{n}, \varphi_{n}\right)<\infty$. But then $u \varphi_{n}$ (which is an element in $\mathcal{F}$ ) also converges to $u$ uniformly on compact sets in $V^{\ell}$, because the function $u$ is bounded. Since $\varphi_{n}$ is bounded by 1 , we find

$$
\begin{aligned}
\mathcal{E}\left(u \varphi_{n}, u \varphi_{n}\right)= & \iint_{x \neq y}\left(u(x) \varphi_{n}(x)-u(y) \varphi_{n}(y)\right)^{2} \mu(x, d y) d x \\
\leq & 2\|u\|_{L^{\infty}}^{2} \iint_{x \neq y}\left(\varphi_{n}(x)-\varphi_{n}(y)\right)^{2} \mu(x, d y) d x \\
& +2 \iint_{x \neq y}(u(x)-u(y))^{2} \mu(x, d y) d x \\
= & 2\|u\|_{L^{\infty}}^{2} \mathcal{E}\left(\varphi_{n}, \varphi_{n}\right)+2 \mathcal{E}(u, u) .
\end{aligned}
$$

This means that $\mathcal{E}\left(u \varphi_{n}, u \varphi_{n}\right)$ is uniformly bounded. Thus, the sequence

$$
\bar{w}_{n}(x, y):=u(x) \varphi_{n}(x)-u(y) \varphi_{n}(y), \quad x, y \in \mathbb{R}^{d}
$$

is a bounded sequence in $L^{2}\left(\mathbb{R}^{d} \times \mathbb{R}^{d} \backslash \Delta ; \mu(x, d y) d x\right)$. An application of the Banach-Saks theorem shows that there is a subsequence $\left\{\bar{w}_{n(k)}\right\}_{k \in \mathbb{N}}$ such that the convex combinations $k^{-1} \sum_{\ell=1}^{k} \bar{w}_{n(\ell)}$ converge to some element $\bar{w} \in L^{2}\left(\mathbb{R}^{d} \times \mathbb{R}^{d} \backslash \Delta\right.$; $\mu(x, d y) d x)$. On the other hand, the sequence $\left\{k^{-1} \sum_{\ell=1}^{k} \varphi_{n(\ell)}\right\}_{k \in \mathbb{N}}$ converges to 1 uniformly on compact sets in $V^{\ell}$, and we know that for $\mu(x, d y) d x$-a.a. $(x, y) \in \mathbb{R}^{d} \times \mathbb{R}^{d} \backslash \Delta$,

$$
\begin{aligned}
\frac{1}{k} \sum_{\ell=1}^{k} \bar{w}_{n(\ell)}(x, y) & =\frac{1}{k} \sum_{\ell=1}^{k}\left(u(x) \varphi_{n(\ell)}(x)-u(y) \varphi_{n(\ell)}(y)\right) \\
& =u(x)\left(\frac{1}{k} \sum_{\ell=1}^{k} \varphi_{n(\ell)}(x)\right)-u(y)\left(\frac{1}{k} \sum_{\ell=1}^{k} \varphi_{n(\ell)}(y)\right) .
\end{aligned}
$$


Therefore, we get, for any compact set $K \subset V^{\ell}$,

$$
\begin{aligned}
\iint_{K \times K \backslash \Delta} & (\bar{w}(x, y)-(u(x)-u(y)))^{2} \mu(x, d y) d x \\
= & \iint_{K \times K \backslash \Delta}\left(\bar{w}(x, y)-\lim _{k \rightarrow \infty} \frac{1}{k} \sum_{\ell=1}^{k} \bar{w}_{n(\ell)}(x, y)\right)^{2} \mu(x, d y) d x \\
= & \lim _{k \rightarrow \infty} \iint_{K \times K \backslash \Delta}\left(\bar{w}(x, y)-\frac{1}{k} \sum_{\ell=1}^{k} \bar{w}_{n(\ell)}(x, y)\right)^{2} \mu(x, d y) d x \\
\leq & \lim _{k \rightarrow \infty} \iint_{\mathbb{R}^{d} \times \mathbb{R}^{d} \backslash \Delta}\left(\bar{w}(x, y)-\frac{1}{k} \sum_{\ell=1}^{k} \bar{w}_{n(\ell)}(x, y)\right)^{2} \mu(x, d y) d x=0
\end{aligned}
$$

where the second equality follows from dominated convergence. Since $\ell \in \mathbb{N}$ was arbitrary, this estimate proves that

$$
\bar{w}(x, y)=u(x)-u(y)=: \bar{u}(x, y) \quad \mu(x, d y) d x \text {-a.e. }
$$

Set $\psi_{k}(x)=u(x) \cdot\left(k^{-1} \sum_{\ell=1}^{k} \varphi_{n(\ell)}(x)\right), x \in \mathbb{R}^{d}$, for each $k$. Then $\psi_{k} \in \mathcal{F}$ and $\psi_{k}$ converges to $u$ a.e. In order to show that $u \in \mathcal{F}_{e}$ it is enough to prove that $\left\{\psi_{k}\right\}_{k \in \mathbb{N}}$ is an $\sqrt{\mathcal{E}}$-Cauchy sequence. Now

$$
\begin{aligned}
\mathcal{E}\left(\psi_{k}-\psi_{k^{\prime}}, \psi_{k}-\psi_{k^{\prime}}\right)= & \iint_{x \neq y}\left(\left(\psi_{k}(x)-\psi_{k^{\prime}}(x)\right)-\left(\psi_{k}(y)-\psi_{k^{\prime}}(y)\right)\right)^{2} \mu(x, d y) d x \\
= & \iint_{x \neq y}\left(\frac{1}{k} \sum_{\ell=1}^{k} \bar{w}_{n(\ell)}(x, y)-\frac{1}{k^{\prime}} \sum_{\ell=1}^{k^{\prime}} \bar{w}_{n(\ell)}(x, y)\right)^{2} \mu(x, d y) d x \\
\leq & \left(\sqrt{\iint_{x \neq y}\left(\frac{1}{k} \sum_{\ell=1}^{k} \bar{w}_{n(\ell)}(x, y)-\bar{u}(x, y)\right)^{2} \mu(x, d y) d x}\right. \\
& \left.+\sqrt{\iint_{x \neq y}\left(\bar{u}(x, y)-\frac{1}{k^{\prime}} \sum_{\ell=1}^{k^{\prime}} \bar{w}_{n(\ell)}(x, y)\right)^{2} \mu(x, d y) d x}\right)^{2} \\
& \stackrel{k, k^{\prime} \rightarrow \infty}{\longrightarrow} 0 .
\end{aligned}
$$

This proves $u \in \mathcal{F}_{e}$.

Theorem 3.2 can also be shown by using a recent characterization of reflected Dirichlet spaces due to Chen and Fukushima [CF11].

As usual we write $u \in \mathcal{F}_{\text {loc }}$ if for every relatively compact, open set $G \Subset \mathbb{R}^{d}$ there exists some $u_{G} \in \mathcal{F}$ such that $\left.u\right|_{G}=\left.u_{G}\right|_{G}$. Using the Beurling-Deny representation of the (quasi-)regular Dirichlet form $(\mathcal{E}, \mathcal{F})$ we can extend $\mathcal{E}$ to $\mathcal{F}_{\text {loc }}$ 
by

$$
\tilde{\mathcal{E}}(u, u):=\sup _{G \Subset \mathbb{R}^{d}} \frac{1}{2} \iint_{G \times G \backslash \Delta}\left(u_{G}(x)-u_{G}(y)\right)^{2} N(d x, d y)
$$

and we define the reflected Dirichlet space as

$$
\mathcal{F}^{\text {ref }}:=\left\{u: \mathbb{R}^{d} \stackrel{\text { measurable }}{\longrightarrow} \mathbb{R}: \begin{array}{c}
\Theta_{k} u \in \mathcal{F}_{\text {loc }} \text { for each } k \in \mathbb{N} \\
\text { and } \sup _{k} \tilde{\mathcal{E}}\left(\Theta_{k} u, \Theta_{k} u\right)<\infty
\end{array}\right\},
$$

where $\Theta_{k} u:=(-k) \vee(u \wedge k)$.

Fukushima and Chen (compare also Chen [Ch92] and Kuwae [Kuw02] for related, less complete results) prove in [CF11] that for recurrent (quasi-)regular Dirichlet spaces $\mathcal{F}^{\text {ref }}=\mathcal{F}_{e}$; their method uses techniques from stochastic analysis, it is less direct than our approach, but also more general.

Lemma 3.3. Assume that $\Phi \in L_{\mathrm{loc}}^{1}, \Psi \in L^{\infty}$ and that the kernel $\nu$ is locally shift-bounded. Then $\mathcal{F}^{\mathrm{ref}}=\dot{\mathcal{D}}[\mathcal{E}]$.

Proof. Under our assumptions, Theorem 2.4 shows that $(\mathcal{E}, \mathcal{F})$ is regular. This means that $\mathcal{F}^{\text {ref }}$ is well-defined.

Since $\Theta_{k}(x)=(-k) \vee x \wedge k$ is a normal contraction, we find for $u_{k}:=\Theta_{k} u$ that $\tilde{\mathcal{E}}\left(u_{k}, u_{k}\right) \leq \tilde{\mathcal{E}}\left(u_{k+1}, u_{k+1}\right)$ and $\sup _{k \in \mathbb{N}} \tilde{\mathcal{E}}\left(u_{k}, u_{k}\right)=\lim _{k \rightarrow \infty} \tilde{\mathcal{E}}\left(u_{k}, u_{k}\right)$. If we use Lebesgue's convergence theorem on the product space $(G \times G \backslash \Delta, N(d x, d y))$ and for any relatively compact open set $G$, we see that $\tilde{\mathcal{E}}(u, u)=\lim _{k \rightarrow \infty} \tilde{\mathcal{E}}\left(u_{k}, u_{k}\right)$ and $\mathcal{F}^{\mathrm{ref}} \subset \dot{\mathcal{D}}[\mathcal{E}]$.

Note that this inclusion depends on the representation (1.1) of $\mathcal{E}$ nor on $\Phi \in$ $L_{\text {loc }}^{1}$ and $\Psi \in L^{\infty}$.

Now assume that $u \in \dot{\mathcal{D}}[\mathcal{E}]$ and choose for a fixed relatively compact set $G \Subset \mathbb{R}^{d}$ some $\phi=\phi_{G} \in C_{0}^{\infty}\left(\mathbb{R}^{d}\right)$ such that $\mathbb{1}_{G} \leq \phi \leq 1$. Then we see as in the proof of Theorem 2.5 that for each $k \in \mathbb{N}$ and $u_{k}:=\Theta_{k} u$,

$$
\begin{aligned}
\mathcal{E}\left(J_{\varepsilon}\left[u_{k} \phi\right], J_{\varepsilon}\left[u_{k} \phi\right]\right) & \leq c \mathcal{E}\left(u_{k} \phi, u_{k} \phi\right)+4 k^{2}\|\Psi\|_{L^{\infty}} \\
& \leq 2 c \mathcal{E}\left(u_{k}, u_{k}\right)+2 c k^{2} \mathcal{E}(\phi, \phi)+4 k^{2}\|\Psi\|_{L^{\infty}} \\
& \leq 2 c\left(\mathcal{E}(u, u)+k^{2} \mathcal{E}(\phi, \phi)+2 k^{2}\|\Psi\|_{L^{\infty}}\right) .
\end{aligned}
$$

This means that the family $\left\{J_{\varepsilon}\left[u_{k} \phi\right]\right\}_{\varepsilon>0}$ is $\mathcal{E}_{1}$-bounded and, as in the proof of Theorem 2.4, we find a subsequence $\left\{J_{\varepsilon(\ell)}\left[u_{k} \phi\right]\right\}_{\ell \in \mathbb{N}}$ whose Cesàro means converge in $\sqrt{\mathcal{E}_{1}}$ to $u_{k} \phi$. This shows that $\Theta_{k} u \cdot \phi \in \mathcal{F}$, hence $u \in \mathcal{F}^{\text {ref }}$. Consequently, $\dot{\mathcal{D}}[\mathcal{E}] \subset \mathcal{F}^{\text {ref }}$, and we see $\dot{\mathcal{D}}[\mathcal{E}]=\mathcal{F}^{\mathrm{ref}}$. 


\section{§4. The active reflected Dirichlet space and its extension}

We will finally study the relation between active reflected Dirichlet spaces and the associated extended reflected spaces. We still assume that $(\mathcal{E}, \mathcal{F})$ is a pure jump Dirichlet form on $L^{2}\left(\mathbb{R}^{d}, m\right)$ with representation (1.1). It is, however, easy to adapt the definition of the extension $\tilde{\varepsilon}$ (just before Lemma 3.3) to the general case including local and killing parts; since this adds nothing new, we refrain from doing so. Let us, nevertheless, begin with an example in the local case. Recall that

$$
\mathcal{F}_{a}^{\mathrm{ref}}:=\mathcal{F}^{\mathrm{ref}} \cap L^{2}\left(\mathbb{R}^{d}\right)
$$

is the active reflected Dirichlet space (cf. Chen [Ch92]).

Example 4.1 (Chen and Fukushima [CF09]). Consider Brownian motion in $\mathbb{R}^{3}$, its generator $\frac{1}{2} \Delta$ and the classical Dirichlet integral $\mathcal{D}(u, u)=\int(\nabla u)^{2} d x$ with Lebesgue measure as reference measure $m$. Recently Chen and Fukushima [CF09] showed the following characterization of the reflected Dirichlet space in terms of the Beppo-Levi space:

$$
\mathcal{F}^{\mathrm{ref}}=B L\left(\mathbb{R}^{3}\right):=\left\{u \in L_{\text {loc }}^{2}\left(\mathbb{R}^{3}\right): \nabla u \in L^{2}\left(\mathbb{R}^{3}\right)\right\} .
$$

By definition, $\mathcal{F}_{a}^{\text {ref }}=\mathcal{F}^{\text {ref }} \cap L^{2}\left(\mathbb{R}^{3}\right)$, which is the Sobolev space $W^{1}\left(\mathbb{R}^{3}\right)=H^{1}\left(\mathbb{R}^{3}\right)$. Since three-dimensional Brownian motion is transient, $\left(H_{e}^{1}\left(\mathbb{R}^{3}\right), \frac{1}{2} \mathcal{D}\right)$ is a Hilbert space; as such it does not contain the function $x \mapsto 1$ while the space $B L\left(\mathbb{R}^{3}\right)$ clearly contains 1 . This shows that, in general, $\left(\mathcal{F}_{a}^{\text {ref }}\right)_{e} \subsetneq \mathcal{F}^{\text {ref }}$.

We will see that Example 4.1 is typical in the sense that one needs some additional condition to ensure $\left(\mathcal{F}_{a}^{\text {ref }}\right)_{e}=\mathcal{F}^{\text {ref }}$.

Note that $\mathcal{F}_{\text {loc }}$ is a lattice: fix $G \Subset \mathbb{R}^{d}$ and $u, v \in \mathcal{F}_{\text {loc }}$. If $u_{G}, v_{G} \in \mathcal{F}$ with $\left.u\right|_{G}=\left.u_{G}\right|_{G}$ and $\left.v\right|_{G}=\left.v_{G}\right|_{G}$ then $u_{G} \wedge v_{G} \in \mathcal{F}$ and

$$
\left.\left(u_{G} \wedge v_{G}\right)\right|_{G}=\left.\left.u_{G}\right|_{G} \wedge v_{G}\right|_{G}=\left.\left.u\right|_{G} \wedge v\right|_{G}=\left.(u \wedge v)\right|_{G} .
$$

Moreover, we find, for $u, v \in \mathcal{F}^{\text {ref }}$,

$$
\begin{aligned}
& \tilde{\mathcal{E}}(u+v, u+v)=\tilde{\mathcal{E}}((u \vee v)+(u \wedge v),(u \vee v)+(u \wedge v)), \\
& \tilde{\mathcal{E}}(u-v, u-v) \geq \tilde{\mathcal{E}}(|u-v|,|u-v|)=\tilde{\mathcal{E}}((u \vee v)-(u \wedge v),(u \vee v)-(u \wedge v))
\end{aligned}
$$

Expanding the expression on either side and adding the resulting (in)equalities yields

$$
\tilde{\mathcal{E}}(u, u)+\tilde{\mathcal{E}}(v, v) \geq \tilde{\mathcal{E}}(u \wedge v, u \wedge v)+\tilde{\mathcal{E}}(u \vee v, u \vee v) \geq \tilde{\mathcal{E}}(u \wedge v, u \wedge v)
$$

This shows that $\mathcal{F}^{\text {ref }}$ is a lattice too. 
Clearly, $\left(\tilde{\mathcal{E}}, \mathcal{F}_{a}^{\text {ref }}=\mathcal{F}^{\text {ref }} \cap L^{2}\left(\mathbb{R}^{d}\right)\right)$ is a Dirichlet form extending $(\mathcal{E}, \mathcal{F})$; we denote by $\tilde{A}$ its generator. As usual,

$$
\begin{aligned}
&\left(\mathcal{F}_{a}^{\text {ref }}\right)_{e}:=\left\{u: \mathbb{R}^{d} \stackrel{\text { measurable }}{\longrightarrow} \mathbb{R}: \exists\left(u_{k}\right)_{k \in \mathbb{N}} \subset \mathcal{F}_{a}^{\text {ref }},\right. \\
&\left.u_{k} \stackrel{\text { a.e. }}{\longrightarrow} u \text { and } \lim _{j, k \rightarrow \infty} \tilde{\mathcal{E}}\left(u_{k}-u_{j}, u_{k}-u_{j}\right)=0\right\} \\
&=\left\{u: \mathbb{R}^{d} \stackrel{\text { measurable }}{\longrightarrow} \mathbb{R}: \exists\left(u_{k}\right)_{k \in \mathbb{N}} \subset \mathcal{F}_{a}^{\text {ref }},\right. \\
&\left.u_{k} \stackrel{\text { a.e. }}{\longrightarrow} u \text { and } \sup _{k \in \mathbb{N}} \tilde{\mathcal{E}}\left(u_{k}, u_{k}\right)<\infty\right\},
\end{aligned}
$$

denotes the extended Dirichlet space of $\left(\mathcal{F}_{a}^{\mathrm{ref}}, \tilde{\mathcal{E}}\right)$. The inclusion ' $C$ ' in the second equality is trivial. The converse, ' $\supset$ ' follows from a Banach-Saks argument applied to the seminorm $\tilde{\varepsilon}\left(u_{k}, u_{k}\right)=\left\|(-\tilde{A})^{1 / 2} u_{k}\right\|_{L^{2}}^{2}$.

We can now state the main theorem of this section.

Theorem 4.2. Let $(\mathcal{E}, \mathcal{F})$ be a Dirichlet form and let $\mathcal{F}_{a}^{\mathrm{ref}}$ and $\left(\mathcal{F}_{a}^{\mathrm{ref}}\right)_{e}$ be the active reflected Dirichlet space and the extended active reflected Dirichlet space, respectively. Then $\left(\mathcal{F}_{a}^{\mathrm{ref}}\right)_{e} \subset \mathcal{F}^{\mathrm{ref}}$. $\left(\mathcal{F}_{a}^{\mathrm{ref}}\right)_{e}$.

If $1 \in\left(\mathcal{F}_{a}^{\mathrm{ref}}\right)_{e}$, then the converse inclusion also holds; in particular, $\mathcal{F}^{\mathrm{ref}}=$

If $(\mathcal{E}, \mathcal{F})$ or $\left(\tilde{\mathcal{E}}, \mathcal{F}_{a}^{\text {ref }}\right)$ is recurrent, then $1 \in\left(\mathcal{F}_{a}^{\text {ref }}\right)_{e}$ and $\mathcal{E}(1,1)=0$. This means, in particular, that for recurrent Dirichlet forms the sets $\left(\mathcal{F}_{a}^{\text {ref }}\right)_{e}=\mathcal{F}^{\text {ref }}$ coincide; if we deal with a jump-type Dirichlet form with shift-bounded kernel, then $\left(\mathcal{F}_{a}^{\mathrm{ref}}\right)_{e}=\mathcal{F}^{\mathrm{ref}}=\mathcal{F}_{e}=\dot{\mathcal{D}}[\mathcal{E}]$.

Proof of Theorem 4.2. We begin with the first inclusion. Let $u \in\left(\mathcal{F}_{a}^{\mathrm{ref}}\right)_{e}$ and let $\left(u_{\ell}\right)_{\ell \in \mathbb{N}} \subset \mathcal{F}_{a}^{\text {ref }}$ be any approximating sequence such that $u_{\ell} \underset{\ell \rightarrow \infty}{\stackrel{\text { a.e. }}{\longrightarrow}} u$ and $\sup _{\ell \in \mathbb{N}} \tilde{\varepsilon}\left(u_{\ell}, u_{\ell}\right)<\infty$.

Since $u_{\ell} \in \mathcal{F}^{\text {ref }}$ we see that $\Theta_{k} u_{\ell} \in \mathcal{F}_{\text {loc }}$ for all $k$. Using the contraction property we get $\tilde{\mathcal{E}}\left(\Theta_{k} u_{\ell}, \Theta_{k} u_{\ell}\right) \leq \tilde{\mathcal{E}}\left(u_{\ell}, u_{\ell}\right)$, hence

$$
\sup _{\ell, k \in \mathbb{N}} \tilde{\mathcal{E}}\left(\Theta_{k} u_{\ell}, \Theta_{k} u_{\ell}\right) \leq \sup _{\ell \in \mathbb{N}} \tilde{\mathcal{E}}\left(u_{\ell}, u_{\ell}\right)<\infty .
$$

Therefore, we may use the Banach-Saks theorem to get a subsequence $\left(u_{\ell(j)}\right)_{j \in \mathbb{N}}$ satisfying

$$
\frac{1}{n} \sum_{j=1}^{n} \Theta_{k} u_{\ell(j)} \underset{n \rightarrow \infty}{\stackrel{\tilde{\varepsilon}}{\longrightarrow}} \Theta_{k} u .
$$

In particular, $\Theta_{k} u \in \mathcal{F}_{a}^{\text {ref }}$ and $\Theta_{m} \Theta_{k} u \in \mathcal{F}_{\text {loc }}$ for all $m \in \mathbb{N}$. For $m \geq k$ this shows $\Theta_{k} u \in \mathcal{F}_{\text {loc }}$. 
From (4.2), a convexity argument and (4.1) we also conclude that

$$
\begin{aligned}
\tilde{\mathcal{E}}\left(\Theta_{k} u, \Theta_{k} u\right) & =\lim _{n \rightarrow \infty} \tilde{\mathcal{E}}\left(\sum_{j=1}^{n} \frac{1}{n} \Theta_{k} u_{n(j)}, \sum_{j=1}^{n} \frac{1}{n} \Theta_{k} u_{n(j)}\right) \\
& \leq \lim _{n \rightarrow \infty} \frac{1}{n} \sum_{j=1}^{n} \tilde{\mathcal{E}}\left(\Theta_{k} u_{n(j)}, \Theta_{k} u_{n(j)}\right) \leq \sup _{\ell \in \mathbb{N}} \tilde{\mathcal{E}}\left(u_{\ell}, u_{\ell}\right)<\infty
\end{aligned}
$$

This shows that $u \in \mathcal{F}^{\text {ref }}$ and $\left(\mathcal{F}_{a}^{\text {ref }}\right)_{e} \subset \mathcal{F}^{\text {ref }}$.

Now we assume that $1 \in\left(\mathcal{F}_{a}^{\mathrm{ref}}\right)_{e}$. This means that there exists a sequence $\left(\phi_{k}\right)_{k \in \mathbb{N}} \subset \mathcal{F}_{a}^{\text {ref }}$ with $\phi_{k} \underset{k \rightarrow \infty}{\stackrel{\text { a.e. }}{\longrightarrow}} 1, \phi_{k} \geq 0$ and $\sup _{k \in \mathbb{N}} \tilde{\mathcal{E}}\left(\phi_{k}, \phi_{k}\right)<\infty$.

For $u \in \mathcal{F}^{\text {ref }} \cap L^{\infty}$ we set $\psi_{k}:=\|u\|_{L^{\infty}} \phi_{k}$. Clearly

$$
u^{ \pm} \wedge \psi_{k} \underset{k \rightarrow \infty}{\stackrel{\text { a.e. }}{\longrightarrow}} u^{ \pm} \quad \text { and } \quad u^{ \pm} \wedge \psi_{k} \in L^{2} .
$$

Since $u^{ \pm} \wedge \psi_{k} \in \mathcal{F}_{\text {loc }}$ we conclude that $u^{ \pm} \wedge \psi_{k} \in \mathcal{F}_{a}^{\text {ref }}$. Moreover,

$$
\begin{aligned}
\sup _{k \in \mathbb{N}} \tilde{\mathcal{E}}\left(u^{ \pm} \wedge \psi_{k}, u^{ \pm} \wedge \psi_{k}\right) & \leq \tilde{\mathcal{E}}\left(u^{ \pm}, u^{ \pm}\right)+\sup _{k \in \mathbb{N}} \tilde{\mathcal{E}}\left(\psi_{k}, \psi_{k}\right) \\
& =\tilde{\mathcal{E}}\left(u^{ \pm}, u^{ \pm}\right)+\|u\|_{L^{\infty}}^{2} \tilde{\mathcal{E}}\left(\phi_{k}, \phi_{k}\right)<\infty,
\end{aligned}
$$

which proves that $u^{ \pm} \in\left(\mathcal{F}_{a}^{\text {ref }}\right)_{e}$. Therefore, if $1 \in\left(\mathcal{F}_{a}^{\text {ref }}\right)_{e}$, then

$$
\mathcal{F}^{\mathrm{ref}} \cap L^{\infty} \subset\left(\mathcal{F}_{a}^{\mathrm{ref}}\right)_{e} \subset \mathcal{F}^{\mathrm{ref}} \text { and } \mathcal{F}^{\mathrm{ref}} \cap L^{\infty}=\left(\mathcal{F}_{a}^{\mathrm{ref}}\right)_{e} \cap L^{\infty} .
$$

If $u \in \mathcal{F}^{\text {ref }}$ then $\Theta_{\ell} u \in \mathcal{F}^{\text {ref }} \cap L^{\infty}$ (note that $\mathcal{F}_{\text {loc }}$ is a lattice), and consequently $\Theta_{\ell} u \in\left(\mathcal{F}_{a}^{\mathrm{ref}}\right)_{e}$. Moreover,

$$
\Theta_{\ell} u \underset{\ell \rightarrow \infty}{\stackrel{\text { a.e. }}{\longrightarrow}} u \text { and } \sup _{\ell \in \mathbb{N}} \tilde{\mathcal{E}}\left(\Theta_{\ell} u, \Theta_{\ell} u\right) \leq \tilde{\mathcal{E}}(u, u)<\infty
$$

which means that $u \in\left(\mathcal{F}_{a}^{\mathrm{ref}}\right)_{e}$. This finally proves $\mathcal{F}^{\mathrm{ref}} \subset\left(\mathcal{F}_{a}^{\mathrm{ref}}\right)_{e}$.

\section{$\S 5$. Appendix}

For the benefit of the readers we summarize in this appendix some basic definitions and notions on Dirichlet forms which have been used in the previous sections.

Let $(X, d)$ be a locally compact separable metric space and $m$ a positive Radon measure on $X$ with full support. Let $\mathcal{F}$ be a dense subspace of $L^{2}(X ; m)$. A symmetric bilinear form $\mathcal{E}$ defined on $\mathcal{F} \times \mathcal{F}$ is called a symmetric Dirichlet form on $L^{2}(X ; m)$ if

(E.1) (nonnegativity) $\mathcal{E}(u, u) \geq 0$ for all $u \in \mathcal{F}$. 
$(\mathcal{E} .2)$ (closedness) $\left(\mathcal{F}, \mathcal{E}_{1}\right)$ is a real Hilbert space with

$$
\mathcal{E}_{1}(u, v):=\mathcal{E}(u, v)+\langle u, v\rangle_{L^{2}(m)}, \quad u, v \in \mathcal{F},
$$

where $\langle u, v\rangle_{L^{2}(m)}$ denotes the inner product of $u$ and $v$ in $L^{2}(X ; m)$.

(E.3) (Markov property) If $u \in \mathcal{F}$, then $v:=0 \vee u \wedge 1 \in \mathcal{F}$ and $\mathcal{E}(v, v) \leq \mathcal{E}(u, u)$.

For a symmetric Dirichlet form $(\mathcal{E}, \mathcal{F})$, there exists a unique non-positive selfadjoint operator $A$ such that

$$
\mathcal{E}(u, v)=\langle\sqrt{-A} u, \sqrt{-A} v\rangle_{L^{2}(m)}, \quad u, v \in \mathcal{F},
$$

and the semigroup $\left\{e^{t A}: t>0\right\}$ generated by $A$ is a Markov semigroup, i.e.,

$$
0 \leq e^{t A} u \leq 1 \quad \text { whenever } \quad u \in L^{2}(X ; m), \quad 0 \leq u \leq 1 .
$$

The Dirichlet form $(\mathcal{E}, \mathcal{F})$ is said to be regular if $C_{0}(X) \cap \mathcal{F}$ is dense in $C_{0}(X)$ - the space of compactly supported, continuous functions on $X$-with respect to the uniform norm and dense in $\mathcal{F}$ with respect to the Hilbert norm $\sqrt{\mathcal{E}_{1}(\cdot, \cdot)}$.

The Beurling-Deny decomposition ([FOT94, Theorem 3.2.1]) says that any regular symmetric Dirichlet form $(\mathcal{E}, \mathcal{F})$ can be expressed for $u, v \in C_{0}(X) \cap \mathcal{F}$ as follows:

$$
\begin{aligned}
\mathcal{E}(u, v)= & \mathcal{E}^{(c)}(u, v)+\int_{X} u(x) v(x) k(d x) \\
& +\iint_{x \neq y}(u(x)-u(y))(u(x)-u(y)) J(d x, d y) .
\end{aligned}
$$

Here $\mathcal{E}^{(c)}$ is a symmetric form with domain $\mathcal{D}\left[\mathcal{E}^{(c)}\right]=\mathcal{F} \cap C_{0}(X)$ satisfying the strong local property: $\mathcal{E}^{(c)}(u, v)=0$ for $u \in \mathcal{D}\left[\mathcal{E}^{(c)}\right]$ and all $v \in \mathcal{F}$ which are constant on a neighbourhood of $\operatorname{supp} u$, the support of $u$. $J$ is symmetric positive Random measure on $X \times X \backslash \Delta$ and $k$ is a positive Radon measure on $X$. Note that $\mathcal{E}^{(c)}, J$ and $k$ are uniquely determined by $\mathcal{E}$. We call $J$ the jumping measure and $k$ the killing measure of $\mathcal{E}$.

Fukushima's existence theorem shows that, for a regular symmetric Dirichlet form $(\mathcal{E}, \mathcal{F})$, there exists an $m$-symmetric Hunt process $\mathrm{M}=\left(X_{t}, \mathbb{P}_{x}\right)$ on $X$ whose transition function defines a semigroup on $L^{2}(X ; m)$ such that

$$
e^{t A} u(x)=\mathbb{E}_{x}\left[u\left(X_{t}\right)\right] \quad m \text {-a.e. for all } u \in L^{2}(E ; m) \cap \mathcal{B}(X), t>0 .
$$

Here $C(X)$ (resp. $\mathcal{B}(X)$ ) denotes the set of continuous (resp. Borel) functions on $X$. $\mathrm{M}$ is unique up to an appropriate equivalence (see [FOT94] for more information). The jumping measure $J$ explains size and intensity of the jumps of the sample paths, while the measure $k$ governs the killing of the sample paths inside $X$ (cf. [FOT94, Theorem 4.5.2]). 
Example 5.1. Let $X=(M, g)$ be a smooth Riemannian manifold and consider a regular symmetric Dirichlet form $(\mathcal{E}, \mathcal{F})$ defined by

$$
\left\{\begin{array}{l}
\mathcal{E}(u, v)=\int_{M}\langle\operatorname{grad} u, \operatorname{grad} v\rangle d v_{g} \\
\mathcal{F} \text { is the closure of } C_{0}^{\infty}(M) \text { under } \mathcal{E}_{1} .
\end{array}\right.
$$

If $M$ is complete, it is well-known that the Laplace-Beltrami operator, the generator of the form, is essentially self-adjoint on $C_{0}^{\infty}(M)$ (see [D]).

In general we do not know whether the domain of the generator contains nice functions like $C_{0}^{\infty}(M)$-not even for regular, strongly local, symmetric Dirichlet forms. Therefore, we cannot discuss the extensions of Dirichlet forms in the context of self-adjoint extensions.

Given a regular symmetric Dirichlet form $(\mathcal{E}, \mathcal{F})$, we can consider the following class of extensions:

$$
\mathcal{A}_{\mathcal{S}}(\mathcal{E}, \mathcal{F}):=\left\{(\tilde{\mathcal{E}}, \tilde{\mathcal{F}}): \begin{array}{l}
(\tilde{\mathcal{E}}, \tilde{\mathcal{F}}) \text { is a Dirichlet form with } \tilde{\mathcal{F}} \supset \mathcal{F}, \tilde{\mathcal{E}}(u, u)=\mathcal{E}(u, u) \\
\text { for } u \in \mathcal{F}, \text { and } u \cdot v \in \mathcal{F}_{b} \text { whenever } u \in \tilde{\mathcal{F}}_{b} \text { and } v \in \mathcal{F}_{b}
\end{array}\right\}
$$

where $\mathcal{F}_{b}$ (resp. $\left.\tilde{\mathcal{F}}_{b}\right)$ means $\mathcal{F} \cap L^{\infty}(X ; m)$ (resp. $\tilde{\mathcal{F}} \cap L^{\infty}(X ; m)$ ). This class is introduced by Silverstein in [Sil74a, Sil74b] in order to classify Markov processes which are the extensions of the Markov process associated with the Dirichlet form $(\mathcal{E}, \mathcal{F})$. We call an element of $\mathcal{A}_{\mathcal{S}}(\mathcal{E}, \mathcal{F})$ an extension of $(\mathcal{E}, \mathcal{F})$ in Silverstein's sense. For the precise meaning of this extension, see Theorem 20.1 in [Sil74b] or A.4.4 in [FOT94].

\section{Acknowledgements}

Professor M. Fukushima (Osaka) and Professor Z.-Q. Chen (Seattle) drew our attention to the interesting connection of extended and active reflected Dirichlet spaces which led to the current form of Section 4 . We are very grateful for the intensive discussions on these topics and we thank both of them for the possibility to consult preprints of the recently published paper [CF09] and monograph [CF11]. We would like to thank an anonymous referee whose comments helped to improve the exposition of our paper.

Financial support through the JSPS-DFG Cooperation Project Dirichlet Forms, Stochastic Analysis and Interacting Systems (R.S.) and Grant-in-aid for scientific research $(C)$ by MEXT (T.U.) is gratefully acknowledged. 


\section{References}

[AH] D. R. Adams and L. I. Hedberg, Function spaces and potential theory, Springer, 1999 (corr. 2nd printing). Zbl 0834.46021 MR 1411441

[BF] C. Berg and G. Forst, Potential theory on locally compact abelian groups, Springer, 1975. Zbl 0308.31001 MR 0481057

[BBC03] K. Bogdan, K. Burdzy and Z.-Q. Chen, Censored stable processes, Probab. Theory Related Fields 127 (2003), 89-152. Zbl 1032.60047 MR 2006232

[Ch92] Z.-Q. Chen, On reflected Dirichlet spaces, Probab. Theory Related Fields 94 (1992), 135-162. Zbl 0767.60073 MR 1191106

[CF09] Z.-Q. Chen and M. Fukushima, On unique extension of time-change reflecting Brownian motions, Ann. Inst. H. Poincaré Probab. Statist. 45 (2009), 864-875. Zbl 1189.60141 MR 2548508

[CF11] Symmetric Markov processes, time change, and boundary theory, Princeton Univ. Press, 2011. Zbl pre05982635 MR 2849840

[D] E. B. Davies, Heat kernels and spectral theory, Cambridge Univ. Press, 1989. Zbl 0699.35006 MR 0990239

[EK] S. E. Ethier and T. G. Kurtz, Markov processes: characterization and convergence, Wiley, 1986. Zbl 0592.60049

[FOT94] M. Fukushima, Y. Oshima and M. Takeda, Dirichlet forms and symmetric Markov processes, 2nd ed., de Gruyter, 2011. Zbl pre05835825 MR 2778606

[K] O. Kallenberg, Foundations of modern probability, Springer, 2001. Zbl 0996.60001 MR 1876169

[KaT96] T. Kawabata and M. Takeda, On uniqueness problem for local Dirichlet forms, Osaka J. Math. 33 (1996), 881-893. Zbl 0870.31014 MR 1435459

[Kuw02] K. Kuwae, Reflected Dirichlet forms and the uniqueness of Silverstein's extension, Potential Anal. 16 (2002), 221-247. Zbl 0998.31006 MR 1885761

[RZ] M. Röckner and T. S. Zhang, Uniqueness of generalized Schrödinger operators, Part II, J. Funct. Anal. 119 (1994), 455-467. Zbl 0799.35053 MR 1261099

[R] R. L. Royden, Real analysis, 3rd ed., Macmillan, 1988. Zbl 0704.26006 MR 1013117

[SU07] R. L. Schilling and T. Uemura, On the Feller property of Dirichlet forms generated by pseudo differential operators, Tohoku Math. J. 59 (2007), 401-422. Zbl 1141.31006 MR 2365348

[Sil74a] M. L. Silverstein, The reflected Dirichlet space, Illinois J. Math. 18 (1974), 310-355. Zbl 0303.60066 MR 0356253

[Sil74b] _ Symmetric Markov processes, Lecture Notes in Math. 426, Springer, 1974. Zbl 0296.60038 MR 0386032

[St70] E. M. Stein, Singular integrals and differentiability properties of functions, Princeton Univ. Press, 1970. Zbl 0207.13501 MR 0290095

[T96] M. Takeda, Two classes of extensions for generalized Schrödinger operators, Potential Anal. 5 (1996), 1-13. Zbl 0854.31005 MR 1373827

[U04] T. Uemura, On symmetric stable-like processes: some path properties and generators, J. Theor. Probab. 17 (2004), 541-555. Zbl 1067.60020 MR 2091550

[U07] , On an extension of jump-type symmetric Dirichlet forms, Electron. Comm. Probab. 12 (2007), 57-65. Zbl 1139.31003 MR 2300215 